\title{
Management of oncology patients receiving anti- cancer treatment in the COVID-19 pandemic
}

Esat Namal ( $\sim$ esatnamal2000@yahoo.com )

Demiroglu Bilim University

Nur Dinc

Demiroglu Bilim University

Sezer Saglam

Demiroglu Bilim University

Ali Vefa Ozturk

Demiroglu Bilim University

Safiye Koculu

Demiroglu Bilim University

Asiye Yir

Demiroglu Bilim University

Esin Cevik

Demiroglu Bilim University

Soheyl Sabet

Demiroglu Bilim University

Bedriye Koyuncu Sokmen

Demiroglu Bilim University

Nagihan Inan Gurcan

Demiroglu Bilim University

Yaman Tokat

Demiroglu Bilim University

Cavlan Ciftci

Demiroglu Bilim University

\section{Research Article}

Keywords: COVID-19, pandemic, chemotherapy, oncology

Posted Date: May 15th, 2020

DOI: https://doi.org/10.21203/rs.3.rs-28858/v1 
License: (c) (i) This work is licensed under a Creative Commons Attribution 4.0 International License. Read Full License 


\section{Abstract}

Background/Aim: Severe acute respiratory syndrome coronavirus 2 (SARS CoV-2) has deeply affected life all over the World. The World Health Organization named this disease as COVID-19. The most important factor in the transmission of the disease is asymptomatic carriers. We've tested all oncology patients, that receive anti-cancer therapy, for COVID-19 to prevent asymptomatic oncology patients from spreading infection and to make the decision to postpone chemotherapy in infected patients. Then, we analyzed the clinical and radiological findings of infected patients.

Materials and Methods: Oncology patients who have indications of receiving anti-cancer treatment in the hospital were tested for COVID-19, two day prior to their treatment even if they were asymptomatic by collecting nasopharyngeal and oropharyngeal swab specimens for RT-PCR for viral RNA detection. Positive patients, underwent inspiratory phase of chest computed tomography (CT) examination. Infected patients were given the recommended treatment for COVID-19. Anti-cancer treatment of all patients that had positive PCR results was delayed for 14 days.

Results: PCR test was positive in 28 of 312 patients that we tested, and the positivity rate was $8.9 \%$. Three patients $(10.7 \%)$ had symptoms; 2 of whom had dyspnea and cough, and 1 had headache, and 25 patients $(89.3 \%)$ had no symptoms.

Conclusion: In oncology patients, who are receiving anti-cancer treatment, we have to recognize the asymptomatic COVID-19 infection. We recommend testing for COVID-19 in oncology patients receiving chemotherapy, periodically or before each anti-cancer treatment, in order to continue their treatment without any problems and to prevent the risk of transmission.

\section{Introduction}

Coronoviruses (CoV) are RNA viruses that can be pathogenic in humans and animals. The novel CoV which is called severe acute respiratory syndrome coronavirus 2 (SARS-CoV-2), was identified to transmit from human to human at the end of 2019, and viral pneumonia associated with SARS-CoV-2 started to appear in Wuhan, China [1]. The World Health Organization (WHO) named this disease as COVID-19, which means 2019 CoV disease [2], and declared it to be a pandemic in March 2020.

People infected with COVID-19 can have a wide range of clinical manifestations, from asymptomatic disease to follow-up indication in intensive care unit. The most feared outcome is viral pneumonia, followed by acute respiratory distress syndrome (ARDS) and death. The severe course of the infection is more common in patients with comorbidity and in the elderly. Often person-to-person contamination occurs by inhaling droplets in the air during close contact or by touching the mouth, eyes, or nose after touching an infected surface.

Although the data on the disease have not matured yet, in an analysis in Italy, 20 percent of COVID-19 deaths in the entire country were in cancer patients [3]. In another study conducted in China, 18 of 2007 
COVID-19 infected patients were cancer patients and the infection was reported to cause serious clinical events in cancer patients [4].

During the COVID-19 pandemic, some arrangements were made in the treatment of cancer patients treated in our center in line with the recommendations of international and national cancer associations $[5,6]$. Accordingly, oral treatments were preferred as much as possible, in order to prevent entries of patients to the hospital, and chemotherapy was avoided in patients who were expected to have low survival benefit from treatment. We have shifted nonurgent follow-up visits to telemedicine, via video or phone. Despite these arrangements, all patients who have indications of receiving anti-cancer treatment in the hospital were tested for COVID-19 two day prior to their treatment even if they were asymptomatic, by collecting nasopharyngeal and oropharyngeal swab specimens for reverse-transcription polymerase chain reaction (RT-PCR) for viral RNA detection. The reason for this is to prevent oncology patients, who have to come to the hospital periodically and may be having an asymptomatic infection, from spreading infection and to make the decision to postpone chemotherapy in infected patients. After this screening, we aimed to retrospectively evaluate the clinical and radiological findings of oncology patients determined to be infected with COVID-19 and to contribute to this area with limited data.

\section{Material/methods}

COVID-19 screening was performed on 312 oncology patients that actively receiving anti-cancer treatment by collecting nasopharyngeal and oropharyngeal swab specimens for RT-PCR for viral RNA detection.

28 patients, who were positive RT-PCR results for SARS-CoV-2, underwent inspiratory phase of chest computed tomography (CT) examination with SOMATOM Go Now (Siemens Medical Solutions, Erlangen, Germany). The technical parameters were as following: tube voltage, 100-140 kVp; tube current, 100$250 \mathrm{~mA}$; and tube rotation time, $0.8 \mathrm{~s}$, slice thickness $5 \mathrm{~mm}$, with $1 \mathrm{~mm}$ or $1.5 \mathrm{~mm}$ section thickness for axial, coronal and sagittal reconstructions. All images were assessed by two radiologists (SS, NI) who were blinded to the clinical history and laboratory results. The visual evaluations were determined by the consensus of two radiologists. On visual evaluation, the pattern of the abnormal attenuation as well as the involved lung lobes were recorded. For the quantification, the percentage of involvement lung volume (ILV) occupied by GGO and / or consolidation areas and total lung volume (TLV) were calculated by automatically using commercial software, Myrian® (Intrasense, Montpellier, France). Lung volume (LV) can be measured as lung volume with attenuation from -950-700 HU, the ILV volume can be measured as lung volume with attenuation from $-800-500 \mathrm{HU}$.

Patients who were receiving anti-cancer treatment and who had positive PCR results were routinely given hydroxychloroquine 2x400 mg tablet on the first day for loading, 2x200 mg tablet for maintenance, oseltamivir 2x75 mg tablet, azithromycin 1x500 mg tablet first day and for maintenance 1x250 mg tablet for a total of 5 days. Favipravir 2x1600 mg tablet first dose, and for maintenance $2 \times 600$ mg tablet were 
added to the treatment of one patient requiring hospitalization. The drug was closely monitored for side effects in terms of arrhythmia.

The anti-cancer treatment of all patients that had positive PCR results was delayed for 14 days. At the end of 14 days, patients with two negative PCR tests with an interval of one week were considered as recovered and their anti-cancer treatments continued.

Descriptive statistical data, including mean, standard deviation, median, minimum and maximum were used to describe continuous variables.

The institutional review board of this hospital approved our study, and informed consent was waived due to the retrospective study design.

\section{Results}

COVID-19 PCR test was performed on 312 oncology patients, who were actively receiving anti-cancer therapy at Demiroğlu Bilim University Medical Oncology Clinic, two days prior to the anti-cancer treatment. PCR results of 28 patients (8.9\%) were evaluated as positive, and the clinical, radiological, laboratory and demographic characteristics of these patients were analyzed. 14 of the patients were females and 14 were males. The median age was $57.6 \pm 12.5 .7$ patients were diagnosed with breast, 6 with colon, 5 with lung, 3 with stomach, 2 with ovarian, 2 with pancreatic cancer, 1 with cholangiocarcinoma, 2 with sarcoma (1 patient with Kaposi sarcoma after liver transplant, and 1 patient with liposarcoma). Eleven patients had distant metastases and were receiving palliative therapy, while 17 were receiving adjuvant therapy. There were no patients receiving concurrent radiotherapy. 23 patients had cytotoxic chemotherapy and 5 patients had targeted treatment within a maximum of 3 weeks. Each patient was questioned for symptoms of fever, cough, dyspnea, muscle pain, headache, changes in taste and smell. Three patients (10.7\%) had symptoms; 2 of whom had dyspnea and cough, and 1 had headache, and 25 patients (89.3\%) had no symptoms. As there were patients who were receiving chemotherapy, almost all patients complained of taste disturbance, but this was thought to be due to their oncological treatment. Six patients $(21.4 \%)$ had lymphopenia and three with lymphopenia had elevated crp and one had leukocytosis. Comorbidity was present in $32 \%$ of the patients including diabetes in 2 patients, hypertension in 5 patients, and autoimmune disease in 2 patients (Table 1). In 15 (53.5\%) of 28 patients, the people they lived with at home could not fully comply with social isolation due to compulsory reasons and 12 (42.8\%) patients came to the hospital by using public transport. Anti-cancer treatment was interrupted in all patients.

All of the positive patients received low-dose CT without contrast. 13 (46.4\%) patients had unremarkable chest CT in regard to viral pneumonia, hence 15 (53.6\%) patients showed suggestive findings for COVID19 (Table 1). Ten cases had multifocal involvement while five cases had single lesion. Mean total lung volumes were $4263.73 \pm 1243.26 \mathrm{~mL}$ and $3470.74 \pm 1097.81 \mathrm{~mL}$ in the positive and negative groups, respectively. Mean LV were $2396.41 \pm 771.15 \mathrm{~mL}$ and $1867.17 \pm 535.41 \mathrm{~mL}$, for the right and left lung 
respectively in all cases. The mean of involved Lung Volume (ILV) percentage in patients with suggestive CT findings was $8.9 \% \pm 7.8$.

One patient requiring hospitalization. The patient was intubated 7 days after hospitalization and died after 1 day. Fourteenth day PCR control of 20 patients were negative, no patient had a hospitalization indication except for the patient who died.

\section{Discussion}

SARS CoV-2 has deeply affected life all over the world with its rapid transmission and fatal ARDS. It is stated that the biggest factor in the transmission of the disease is carriers who have the disease asymptomatically. Asymptomatic infection has been reported in the COVID-19 pandemic [7,8]. Although the frequency of asymptomatic infections in society is not fully known, an estimate was made based on the screening during quarantine in the Japanese cruise ship in Yokohama. The cruise ship hosting 3,711 people underwent a 2-week quarantine after a former passenger was found with COVID-19 postdisembarking. 634 people on board tested positive for the causative virus. The researchers conducted statistical modeling to derive the delay-adjusted asymptomatic proportion of infections, along with the infections' timeline. The estimated asymptomatic proportion was 17.9\% [9]. And also, experts from the Infectious Diseases Society of America (IDSA) explain that some of the asymptomatic patients were presymptomatic and started to spread the virus $24-48$ hours before developing symptoms.

Oncology patients have to go to the hospital due to the necessity of continuing their oncological treatment. Although the patients stated that they complied with the rules of isolation in their living spaces, we found that 15 (53.5\%) of 28 patients with whom they live together at home go out to work and $12(42.8 \%)$ patients came to the hospital by using public transport. So they did not fully comply with social isolation due to compulsory reasons. As asymptomatic patients are not tested, the possibility that these patients may be asymptomatic carriers or in presymptomatic period, increases the risk of infection spread to both other patients and healthcare professionals. Therefore, PCR test was performed on every patient who would receive anti-cancer treatment even if they did not have symptoms suggesting COVID19 infection. In our study, PCR test was positive in 28 of 312 patients that we tested, and the positivity rate was $8.9 \%$. Only three $(10.7 \%)$ of the oncology patients had mild symptoms, 25 patients $(89.3 \%)$ had no symptoms.

Because of the changes on computed tomography (CT) of the chest can be positive earlier than other clinical symptoms and laboratory findings, it is very useful for early diagnosis. Common abnormal radiography findings were consolidation and ground glass opacities, with bilateral, peripheral, and lower lung zone distributions [10]. All positive patients underwent a low-dose lung CT, and approximately half of them have COVID-19 involvement. When the limited number of literature is reviewed, a correlation is reported between the level of lung parenchymal involvement in CT and the severity of the clinical course [11]. It is noteworthy that in almost half of our cases there are no CT findings and the involvement in patients with CT findings is relatively limited (approximately 10\%). This finding may explain the low 
frequency of symptoms in the patient group. With the treatment given in the early period, only one of our patients required hospitalization, and the same patient died 1 week after the diagnosis.

It is not clear that, whether $89.3 \%$ of the patients had asymptomatic infection was coincidental or due to the effect of their immunosuppressive disease and treatments. Since the excessive response of the immune system is considered as one of the causes of lung damage [12], impaired immune response in cancer patients may be the reason that the patients were asymptomatic [13]. No patient developed symptoms in 14 days of follow-up, except for three. So these patients are in the position of a carrier for the spread of the infection. We believe that screening tests should be performed at regular intervals and the isolation rules should be applied more strictly in these patients, even if they are asymptomatic before treatment, in order to continue their treatment without any problems and to prevent the risk of transmission. Also, giving chemotherapy to an asymptomatic but infected patient may increase the severity of the course of infection.

In summary, in oncology patients who are receiving active anti-cancer treatment without symptoms and findings suggestive of COVID-19 infection, we have to recognize the asymptomatic group of patients. For this reason, we recommend testing for COVID-19 in oncology patients actively receiving therapy,

periodically or before each anti-cancer treatment. If we are unable to test before each treatment, it would be correct for healthcare professionals and their families to take precautions as if the patient was COVID19 positive, in terms of the risk of contamination of the people they are in contact with.

\section{References}

1. Surveillance case definitions for human infection with novel coronavirus (nCoV) Interim guidance v2 15 January 2020 WHO/2019-nCoV/Surveillance/v2020.2

2. World Health Organization. Director-General's remarks at the media briefing on 2019-nCoV on 11 February 2020. https://www.who.int/dg/speeches/detail/who-director-general-s-remarks-at-themedia-briefing-on-2019-ncov-on-11-february-2020 Accessed on February 12, 2020.

3. Case-Fatality Rate and Characteristics of Patients Dying in Relation to COVID-19 in Italy. Onder G, Rezza G, Brusaferro S. JAMA. 2020; https://doi.org/ 10.1001/jama.2020.4683

4. Liang W, Guan W, Chen R, et al. Cancer patients in SARS-CoV-2 infection: a nationwide analysis in China. Lancet Oncol 2020; 213: 335-337.

5. COVID-19 Patient Care Information. American Society of Clinical Oncology (ASCO). https://www.asco.org/asco-coronavirus-information/care-individuals-cancer-during-covid-19

6. COVID-19 Patient Care Information. Turkish Society of Medical Oncology. https://www.kanser.org/saglik/haberler/covid-19-pandemisinde-hekimlerin-almalari-gereken-kisiselonlemler/2129

7. Chan JF, Yuan S, Kok KH, To KK, Chu H, Yang J, et al. A familial cluster of pneumonia associated with the 2019 novel coronavirus indicating person-to-person transmission: a study of a family cluster. Lancet. 2020;395:514. 
8. World Health Organization. Coronavirus disease 2019 (COVID-19) Situation Report Epub 2020 Jan 24. https://www.who.int/docs/default-source/coronaviruse/situation-reports/20200217-sitrep-28covid-19.pdf?sfvrsn=a19cf2ad_2

9. Mizumoto K, Kagaya K, Zarebski A, Chowell G. Estimating the asymptomatic proportion of coronavirus disease 2019 (COVID-19) cases on board the Diamond Princess cruise ship, Yokohama, Japan, 2020. Euro Surveill 2020; 25.

10. Shi H, Han X, Jiang N, Cao Y, Alwalid O, Gu J, Fan Y, Zheng C. Radiological findings from 81 patients with COVID-19 pneumonia in Wuhan, China: a descriptive study. Lancet Infect Dis. 2020;20:425.

11. Relation Between Chest CT Findings and Clinical Conditions of Coronavirus Disease (COVID-19) Pneumonia. AJR. 2020; https//doi.org/10.2214/AJR.20.22976

12. Xu Z, Shi L, Wang Y, Zhang J, Huang L, Zhang C, et al. Pathological findings of COVID-19 associated with acute respiratory distress syndrome. Lancet Respir Med 2020; published online Feb 18.

13. Schreiber RD, Old LJ, Smyth MJ. Cancer immunoediting: Integrating immunity's roles in cancer suppression and promotion. Science (80-. ). 2011; 331: 1565-70.

\section{Declarations}

Corresponding Author: Esat Namal, nursenertr@hotmail.com, Merkez Mahallesi, Abide-i Hürriyet Caddesi, no:164,34387 Şişli/İstanbul Fax Number: 0902122244982 Telephone Number: 0905079464716

All procedures followed were in accordance with the ethical standards of the responsible committee on human experimentation (institutional and national) and with the Helsinki Declaration of 1964 and its later amendments. Informed consent was obtained from all patients for being included in the study.

There is no conflict of interest.

\section{Table 1}

Table 1. Demographic, clinical, laboratories and radiological features of patients 


\begin{tabular}{ll}
\hline Variables & $\mathrm{n}(\%)$ \\
\hline Sex & 14 \\
Male & 14 \\
Female & $57.6 \pm 12.5$ \\
\hline Age & \\
\hline Primary site of cancer & 7 \\
Breast cancer & 6 \\
Colorectal cancer & 5 \\
Lung cancer & 3 \\
Gastric cancer & 2 \\
Ovarian cancer & 2 \\
Pancreatic cancer & 1 \\
Cholangiocarcinoma & 2 \\
Soft tissue sarcoma & $13(46.4 \%)$ \\
\hline Stage of cancer & $15(53.6 \%)$ \\
Metastatic disease & 17 \\
Non metastatic disease &
\end{tabular}

